\section{Renal denervation for resistant hypertension: the wrong target?}

\author{
Shi-Sheng Zhou and Yiming Zhou
}

We read with interest the article by Gulati et al. (Opinion: The rise, fall, and possible resurrection of renal denervation. Nat. Rev. Cardiol. 13, 238-244; 2016) ${ }^{1}$. The authors discussed the results of the SYMPLICITY HTN trials and concluded that current evidence is insufficient to declare renal denervation a failure for the treatment of resistant hypertension. In their Perspectives article, however, they have not considered the possibility that the current theoretical basis for renal denervation therapy for resistant hypertension might be erroneous.

Based on the finding of increased renal noradrenaline spillover in patients with hypertension, it is widely accepted that there is renal sympathetic hyperactivity in hypertension, which is used as the theoretical basis for renal denervation therapy ${ }^{2}$. However, this basis is questionable because the contribution of platelets to renal noradrenaline spillover has not been taken into account. The platelets are an important factor that determines plasma noradrenaline levels. They collect, store, and degrade (through monoamine oxidase) noradrenaline from plasma, but release undegraded noradrenaline during their activation (FIG. 1). Given that the lifespan of platelets is around 10 days, $\sim 10 \%$ of circulating platelets are destroyed every day. The concentration of noradrenaline is substantially higher in platelets than in plasma, with a ratio of platelet to plasma concentration of 1855:1 (REF. 3). This ratio indicates that intra-organ platelet destruction is an important source of regional noradrenaline spillover.

Patients with hypertension have platelet abnormalities, including increased platelet volume and elevated platelet noradrenaline levels. In vitro evidence has shown that a high diastolic blood pressure correlates with a rapid efflux of noradrenaline from platelets ${ }^{4}$, suggesting an overload of noradrenaline in patients with hypertension. Importantly, increased platelet destruction can be observed in both renal disorders and severe hypertension. The biopsy findings of renal thrombotic microangiopathy in the context of severe hypertension ${ }^{5}$ show evidence of intrarenal platelet destruction. The increased destruction of platelets that have high levels of noradrenaline might subsequently to increased platelet volume. trigger the release of more noradrenaline into the renal circulation. Moreover, hypertension is associated with renal insufficiency, including renal disease and reduced nephron number. As shown in FIG. 1, renal insufficiency might decrease noradrenaline clearance and thus increase the burden on platelets. Indeed, an inverse correlation between estimated glomerular filtration rate and mean platelet volume has been demonstrated ${ }^{6}$. Therefore, an alternative and likely explanation for the increased renal noradrenaline spillover in hypertension is attributable to a combination of renal insufficiency and increased platelet destruction, accounting for the neutral results of the SYMPLICITY HTN-3 study.

Platelet overload or increased platelet volume can be caused by many factors, including $\operatorname{diet}^{7}$, increased sympathetic activity ${ }^{8}$, and renal insufficiency ${ }^{6}$. Among these factors, renal sympathetic denervation has been shown in SYMPLICITY HTN-3 to be ineffective for resistant hypertension, and reduced nephron number, or renal insufficiency, cannot be reversed by medication. By contrast, dietary risk factors are avoidable, and many have been identified. For example, xenobiotics and excess vitamins can disturb noradrenaline degradation $^{9,10}$. Therefore, treatment of hypertension should focus on reducing known dietary risk factors, rather than on renal denervation.
Shi-Sheng Zhou is at the Institute of Basic Medical Sciences, Medical College, Dalian University, Dalian Economic and Technological Development Zone, Dalian 116622, China.

Yiming Zhou is at the Renal Division, Department of Medicine, Brigham and Women's Hospital, Harvard Institutes of Medicine, Harvard Medical School, 75 Francis Street, Boston, Massachusetts 02115, USA.

Correspondence to S.-S.Z. zhouss@ymail.com

\section{doi: 10.1038/nrcardio.2016.94}

Published online 3 June 2016

1. Gulati, R., Raphael, C. E., Negoita, M., Pocock, S. J. $\&$ Gersh, B. J. The rise, fall, and possible resurrection of renal denervation. Nat. Rev. Cardiol. 13 , 238-244 (2016).

2. Grassi, G., Mark, A. \& Esler, M. The sympathetic nervous system alterations in human hypertension Circ. Res. 116, 976-990 (2015)

3. Smith, C. C., Curtis, L. D., Delamothe, A. P., Prichard, B. N. \& Betteridge, D. J. The distribution of catecholamines between platelets and plasma in normal human subjects. Clin. Sci. (Lond.) 69, 1-6 (1985).

4. Mattiasson, I., Mattiasson, B. \& Hood, B. Uptake and efflux of noradrenaline from platelets; a model system for neurogenic mechanisms in essential hypertension. Clin. Sci. (Lond.) 57 (Suppl. 5), 225s-227s (1979)

5. Zhang, B. et al. Renal thrombotic microangiopathies induced by severe hypertension. Hypertens. Res. 31 479-483 (2008)

6. Verdoia, M. et al. Impact of renal function on mean platelet volume and its relationship with coronary artery disease: a single-centre cohort study. Thromb. Res. 141, 139-144 (2016).

7. Coban, E., Yilmaz, A. \& Sari, R. The effect of weight loss on the mean platelet volume in obese patients. Platelets 18, 212-216 (2007)

8. Ozdemir, O. et al. Association between mean platelet volume and autonomic nervous system functions: increased mean platelet volume reflects sympathetic overactivity. Exp. Clin. Cardiol. 9, 243-247 (2004).

9. Zhou, S. S., Zhou, Y. M., Li, D. \& Lun, Y. Z. Dietary methyl-consuming compounds and metabolic syndrome. Hypertens. Res. 34, 1239-1245 (2011).

10. Zhou, S. S. \& Zhou, Y. Excess vitamin intake: an unrecognized risk factor for obesity. World J. Diabetes 5, 1-13 (2014).

\section{Competing interests statement}

The authors declare no competing interests.

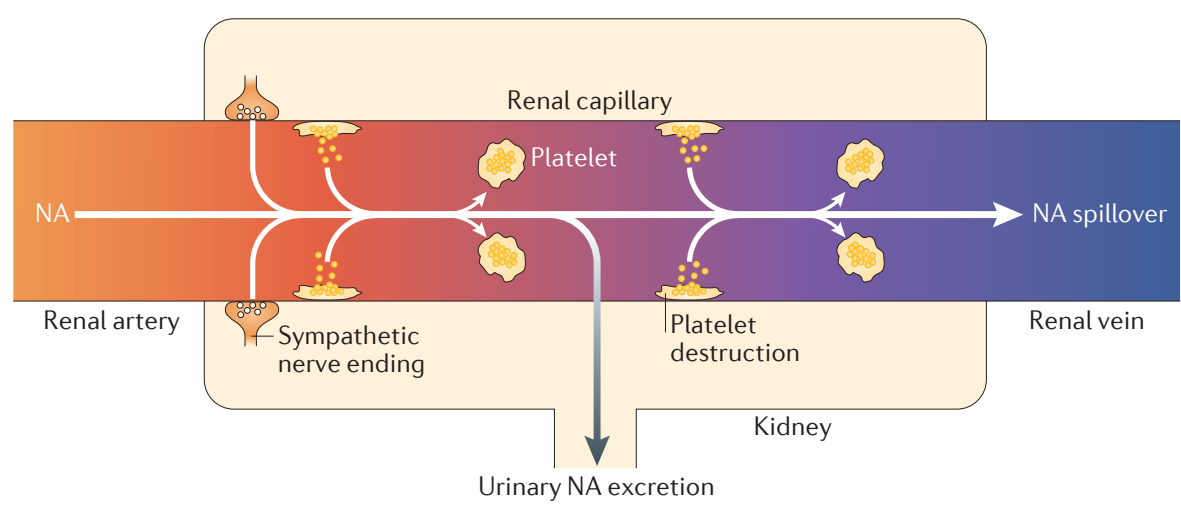

Figure 1 | Factors influencing renal noradrenaline (NA) spillover. The magnitude of renal noradrenaline spillover is determined by sympathetic activity, intrarenal platelet destruction, renal clearance, and platelet uptake. Renal insufficiency increases the burden on platelets, leading 